\title{
Dynamical Casimir Effect Entangles Artificial Atoms
}

\author{
S. Felicetti, ${ }^{1}$ M. Sanz, ${ }^{1}$ L. Lamata, ${ }^{1}$ G. Romero, ${ }^{1}$ G. Johansson,${ }^{2}$ P. Delsing, ${ }^{2}$ and E. Solano ${ }^{1,3}$ \\ ${ }^{1}$ Department of Physical Chemistry, University of the Basque Country UPV/EHU, Apartado 644, E-48080 Bilbao, Spain \\ ${ }^{2}$ Department of Microtechnology and Nanoscience (MC2), Chalmers University of Technology, SE-412 96 Göteborg, Sweden \\ ${ }^{3}$ IKERBASQUE, Basque Foundation for Science, Alameda Urquijo 36, 48011 Bilbao, Spain
}

(Received 2 May 2014; revised manuscript received 9 July 2014; published 27 August 2014)

\begin{abstract}
We show that the physics underlying the dynamical Casimir effect may generate multipartite quantum correlations. To achieve it, we propose a circuit quantum electrodynamics scenario involving superconducting quantum interference devices, cavities, and superconducting qubits, also called artificial atoms. Our results predict the generation of highly entangled states for two and three superconducting qubits in different geometric configurations with realistic parameters. This proposal paves the way for a scalable method of multipartite entanglement generation in cavity networks through dynamical Casimir physics.
\end{abstract}

DOI: 10.1103/PhysRevLett.113.093602

The phenomenon of quantum fluctuations, consisting of virtual particles emerging from vacuum, is central to understanding important effects in nature-for instance, the Lamb shift of atomic spectra [1] and the anomalous magnetic moment of the electron [2]. The appearance of a vacuum-mediated force between two perfectly conducting plates, known as the Casimir effect, is caused by a reduction of the density of electromagnetic modes imposed by the boundary conditions [3-5]. This leads to a vacuum radiation pressure between the mirrors that is lower than the pressure outside. It was also suggested [6] that a mirror undergoing relativistic motion could convert virtual into real photons. This phenomenon, called the dynamical Casimir effect (DCE), has been observed in recent experiments with superconducting circuits $[7,8]$. In the same manner that the Casimir effect can be understood as a mismatch of vacuum modes in space, the kinetic counterpart can be explained as a mismatch of vacuum modes in time.

A moving mirror modifies the mode structure of the electromagnetic vacuum. If the mirror velocity $v$ is much smaller than the speed of light $c$ then the electromagnetic modes adiabatically adapt to the changes and no excitations occur. Otherwise, if the mirror experiences relativistic motion, changes occur nonadiabatically and the field can be excited out of the vacuum, generating real photons. Beyond its fundamental interest, it has been pointed out that the DCE provides a mechanism to generate quantum correlations [9-15]. In this sense, we may consider the study of the DCE as a resource for quantum networks and quantum simulations in the frame of quantum technologies. In circuit quantum electrodynamics, DCE photons have been created by modifying the boundary condition for the electromagnetic field [7]. In a similar experiment, photons have also been created by modulating the effective speed of light [8]. Note that the emergence of the DCE physics in a different quantum platform allows for other geometric
PACS numbers: 42.50.Lc, 03.67.Bg, 84.40.Az, 85.25.Cp

configurations and interaction terms, leading to a variety of different physical conditions.

In this Letter, we investigate how to generate multipartite entangled states of two-level systems, also referred to as quantum bits (qubits), by means of varying boundary conditions in the framework of superconducting circuits. For pedagogical reasons, we illustrate our model with a hypothetical quantum-optical system, shown in Fig. 1. It is composed of two cavities that are coupled to independent single qubits. These cavities share a partially reflecting and transparent mirror, yielding the last interaction term of the Hamiltonian in Eq. (1). We assume that the cavity-qubit coupling strength is much larger than any decoherence rate in the system. In this context, we introduce the key concepts allowing the generation of highly entangled two-qubit states, also known as Bell states [16], in circuit

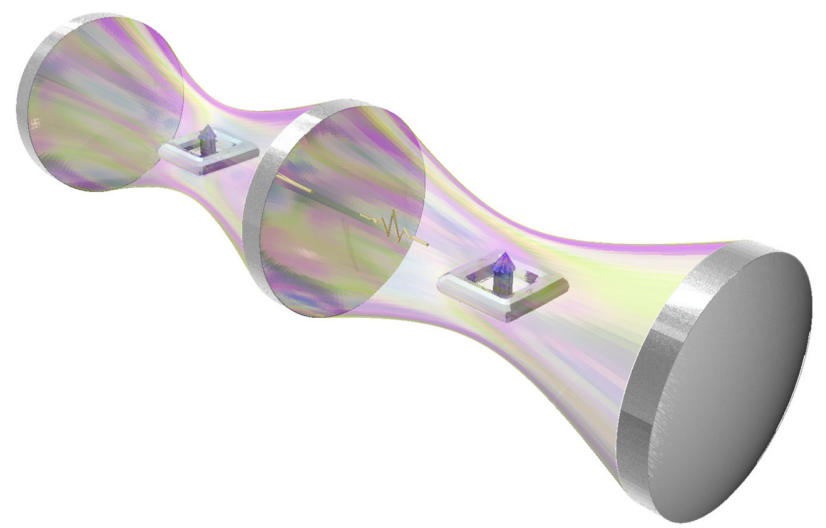

FIG. 1 (color online). Quantum optical implementation of the model of Eq. (1): two cavities with a common partially reflecting mirror, each one containing a two-level artificial atom in the strong-coupling regime. If the position and/or transmission coefficient of the central mirror is time modulated, correlated photon pairs are generated and entanglement is transferred to qubits via the Jaynes-Cummings interaction. 
QED [17-19]. Later, we will consider the generation of tripartite entanglement [20] and the scalability aspects of our proposal to multipartite systems [21].

The Hamiltonian describing the system of Fig. 1 is composed of the sum of two Jaynes-Cummings interactions and a time-dependent coupling between the field quadratures,

$$
\begin{aligned}
\mathcal{H}= & \hbar \sum_{\ell=1}^{2}\left[\omega_{\ell} a_{\ell}^{\dagger} a_{\ell}+\frac{\omega_{\ell}^{q}}{2} \sigma_{\ell}^{z}+g_{\ell}\left(\sigma_{\ell}^{+} a_{\ell}+\sigma_{\ell}^{-} a_{\ell}^{\dagger}\right)\right] \\
& +\hbar \alpha(t)\left(a_{1}^{\dagger}+a_{1}\right)\left(a_{2}^{\dagger}+a_{2}\right) .
\end{aligned}
$$

Here, $a_{\ell}^{\dagger}, a_{\ell}$ are the creation and annihilation operators of the bosonic modes representing the cavity fields, while $\sigma_{\ell}^{z}$, $\sigma_{\ell}^{ \pm}$are the Pauli operators of qubits. The characteristic frequencies of the two cavities are denoted by $\omega_{\ell}$, while the qubit energies are $\omega_{\ell}^{q}$. The parameters $g_{\ell}$ and $\alpha(t)$ denote the cavity-qubit and cavity-cavity interaction strength, respectively.

In Eq. (1), the coupling between different cavity modes, due to the overlap of their spatial distribution, is written in its full form without performing the rotating wave approximation. While in optical cavities this overlap can be obtained with a partially reflecting mirror [24], in circuit QED it is commonly implemented using capacitors or inductances shared by two or more resonators. The boundary condition at the edge shared by the cavities is ruled by the central mirror position and by its reflection coefficient. Modulating these physical quantities results in a time dependence of the cavity frequencies $\omega_{i}$ and of the coupling parameter $\alpha$. When the effective cavity length is oscillating with small deviations from its average value, we can still consider the system as a single-mode resonator. In particular, if the cavity-cavity coupling parameter is a timedependent function, $\alpha(t)=\alpha_{0} \cos \left(\omega_{d} t\right)$ with $\omega_{d}=\omega_{1}+$ $\omega_{2}$ and $\alpha_{0} / \omega_{i} \ll 1$, the interaction effectively turns into a two-mode squeezing term (see below),

$$
\alpha(t) X_{1} X_{2} \rightarrow \frac{\alpha_{0}}{2}\left(a_{1}^{\dagger} a_{2}^{\dagger}+a_{1} a_{2}\right),
$$

which generates pairs of entangled photons shared by the cavities. By means of the Jaynes-Cummings interaction, entanglement generated between cavities may be transferred to resonant qubits. In fact, we will prove below that, under suitably designed conditions, maximal entanglement (Bell state) between the two qubits may be attained.

Nowadays, quantum technologies [25] offer several platforms to study fundamentals and applications of quantum theory. In particular, superconducting circuits technology [26,27] is a prime candidate to implement the model of Eq. (1). In this framework, the cavities are constituted by coplanar waveguides, working at cryogenic temperatures, that are described by an equivalent $L C$ circuit, as shown in Figs. 2(a) and 2(b). The characteristic frequency of such devices is in the $2-10 \mathrm{GHz}$ microwave regime. Each cavity can be coupled to a superconducting qubit built from Josephson junctions (JJs) to access charge [28], flux [29], or phase [30] degrees of freedom. Specifically, we propose the use of transmon qubits which have low sensitivity to charge noise and coherence times well above $10 \mu \mathrm{s}$ [31-33]. The moving mirror [23,34] that couples both cavities (see Fig. 1) can be implemented by means of a superconducting quantum interference device (SQUID) [35], which behaves as a tunable inductance. A SQUID is composed of a superconducting loop interrupted by two JJs [see Fig. 2(a)], threaded by an external flux $\phi_{\text {ext }}$. The latter allows a fast modulation of the electrical boundary condition of cavities and their interaction. Notice that a modulation of the magnetic flux threading the SQUID induces a proportional variation of the effective resonator lengths, while in the system of Fig. 1, moving the central mirror results in an opposite change of cavity lengths.

By using off-the-shelf electronics, it is possible to produce magnetic fluxes that oscillate at the cavity characteristic frequencies. The upper limit to the speed of modulation is imposed by the SQUID plasma frequency, defined as $\omega_{p}=(1 / \hbar) \sqrt{8 E_{C} E_{J}}$, where $E_{C}$ is the charging energy, $E_{J}$ the Josephson energy, both associated with a single JJ belonging to the superconducting loop. Beyond this frequency, the internal degrees of freedom of the device are activated and a more complex behavior appears. The

(a)

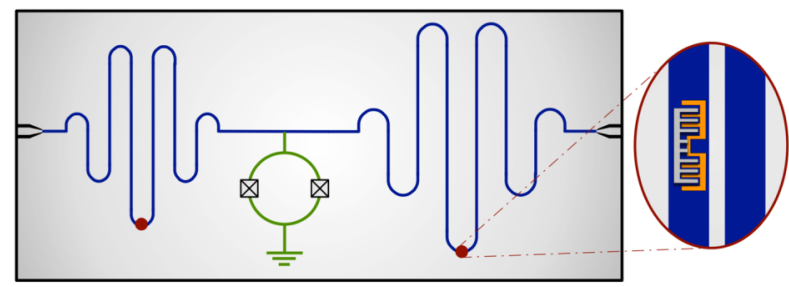

(b)

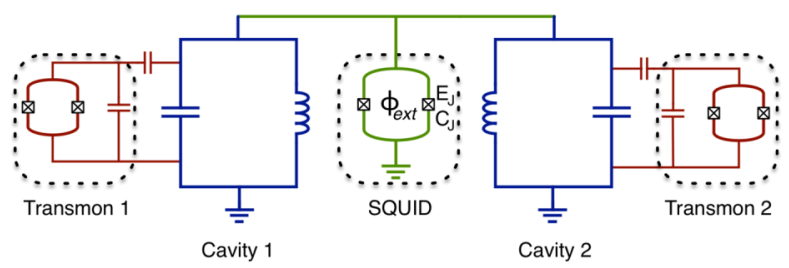

FIG. 2 (color online). (a) The model of Fig. 1 can be implemented by means of two coplanar waveguides, grounded through a SQUID, containing two superconducting qubits. The blue lines represent two parallel strip lines of isolating material, where the superconducting region between them constitutes the coplanar waveguide. Each cavity interacts with a transmon qubit that is denoted by a red dot. Different resonator lengths result in distinct resonator frequencies. (b) Circuit diagram for the previous scheme, where the cavities are effectively represented by $L C$ resonators. We assume two identical Josephson junctions of the SQUID, while transmon qubits are constituted by two Josephson junctions shunted by a large capacitance. 
external flux $\phi_{\text {ext }}(t)$ injected into the device, which also determines $E_{J}$, will be composed of the sum of a signal oscillating at the driving frequency $\omega_{d}$ and a constant offset $\phi_{0}, \quad \phi_{\text {ext }}(t)=\phi_{0}+\Delta \phi \cos \left(\omega_{d} t\right)$. We consider nondegenerate resonators to avoid uncorrelated photon generation at the cavity resonance frequencies, an assumption that has been confirmed by a detailed quantum mechanical analysis of the effective lumped circuit element in Fig. 2(b) [21].

If the instantaneous resonant frequency of a given resonator follows the time dependence $\omega(t)=\omega_{0}+$ $\delta \omega \cos \left(\omega_{d} t\right)$, cavity modes are well defined only under the condition $\delta \omega \ll \omega_{0}$. In our proposal, the frequencies of the cavity modes are obtained by solving the transcendental equation $k d \tan (k d)=L / L_{s}-C_{s} / C(k d)^{2}$ for the wave number $k$, where $d$ is the length of the resonator. We called $C_{s}, L_{s}$, and $C, L$ the effective capacitance and inductance of the SQUID and of the resonator, respectively. Parameters used in our simulations assure that $\delta \omega / \omega_{0}<10^{-3}$.

In the interaction picture, the parametric processes induced by the SQUID lead to the Hamiltonian

$$
\begin{aligned}
\mathcal{H}_{\mathrm{d}}^{I}(t)= & \hbar \cos \left(\phi_{\mathrm{ext}} / \varphi_{0}\right)\left[\sum_{\ell=1}^{2} \alpha_{\ell}\left(a_{\ell} e^{-i \omega_{\ell} t}+a_{\ell}^{\dagger} e^{i \omega_{\ell} t}\right)^{2}\right. \\
& \left.-\hbar \tilde{\alpha}\left(a_{1} e^{-i \omega_{1} t}+a_{1}^{\dagger} e^{i \omega_{1} t}\right)\left(a_{2} e^{-i \omega_{2} t}+a_{2}^{\dagger} e^{i \omega_{2} t}\right)\right],
\end{aligned}
$$

where $\varphi_{0}=\hbar / 2 e$ is the reduced flux quantum, and the coefficients $\alpha_{\ell}$ and $\tilde{\alpha}$ are functions of the Josephson energy $\left(E_{J}\right)$, the junction capacitance $\left(C_{J}\right)$, the cavity parameters such as capacitance $\left(C_{\ell}\right)$ and inductance $\left(L_{\ell}\right)$. If the parameters $\alpha_{\ell}$ and $\tilde{\alpha}$ are much smaller than cavity frequencies $\omega_{\ell}$, we can perform the rotating wave approximation (RWA), and so neglect fast-oscillating terms in Eq. (3). In this case, if we consider $\phi_{\text {ext }}=\phi_{0}+\Delta \phi \cos \left(\omega_{d} t\right)$ with $\Delta \phi$ a small flux amplitude, then controlling the driving frequency $\omega_{d}$ allows us to selectively activate interaction terms in the system dynamics. When the cavity is off resonant and $\omega_{d}=\omega_{1}+\omega_{2}$, the interaction Hamiltonian reads as Eq. (2). Interactions among different cavity modes, called mode mixing, are activated under the frequencymatching condition $\omega_{d}=\omega_{a}-\omega_{b}$. Cavity and driving frequencies can be chosen in order to make the relevant mode interact only with off resonance, overdamped modes. Circuit design allows each qubit to be resonantly coupled with a single cavity mode, in which activation of higher modes due to the DCE mechanism can be neglected.

Our protocol for generating entanglement requires neither direct [36] nor single cavity-bus mediated [37] qubitqubit interaction. Instead, it consists of cooling down the system to its ground state, turning on the external driving flux $\phi_{\text {ext }}$, and switching it off at time $t_{S O}$ when the maximal qubit entanglement is reached. The concurrence $\mathcal{C}$ is an entanglement monotone of a given bipartite mixed state $\rho$, namely, the minimum average entanglement of an ensemble of pure states that represents $\rho$. For an arbitrary two-qubit state the concurrence reads [38] $\mathcal{C}(\rho)=$ $\max \left\{0, \lambda_{1}-\lambda_{2}-\lambda_{3}-\lambda_{4}\right\}$, where $\lambda_{i}$ are the eigenvalues, in decreasing order, of the Hermitian matrix $R=$ $\sqrt{\sqrt{\rho} \tilde{\rho} \sqrt{\rho}}$, with $\tilde{\rho}=\sigma_{y} \otimes \sigma_{y} \rho^{*} \sigma_{y} \otimes \sigma_{y}$.

The numerical results are shown in Fig. 3(a). An almost maximally entangled state $(\mathcal{C}=0.97)$ can be reached within $t_{S O} \approx 10-500 \mathrm{~ns}$, that is, for a wide range of realistic system parameters [21]. Such protocol allows generation of the Bell state $|\psi\rangle=(|e e\rangle+i|g g\rangle) / \sqrt{2}$ with fidelity $\mathcal{F}=|\langle\psi|\rho| \psi\rangle|=0.99$, with current superconducting circuits technology. The density matrix of the produced Bell state is shown in Fig. 3(b). We have also proven that entanglement generation is robust against small imperfections due to limited fabrication precision and imperfect ground-state preparation. Our protocol can be implemented in an on-chip architecture and it does not require any external source of squeezed signals [39].

In the framework of superconducting circuits, resonators can be linked together in unidimensional and bidimensional arrays to build networks of quantum cavities and
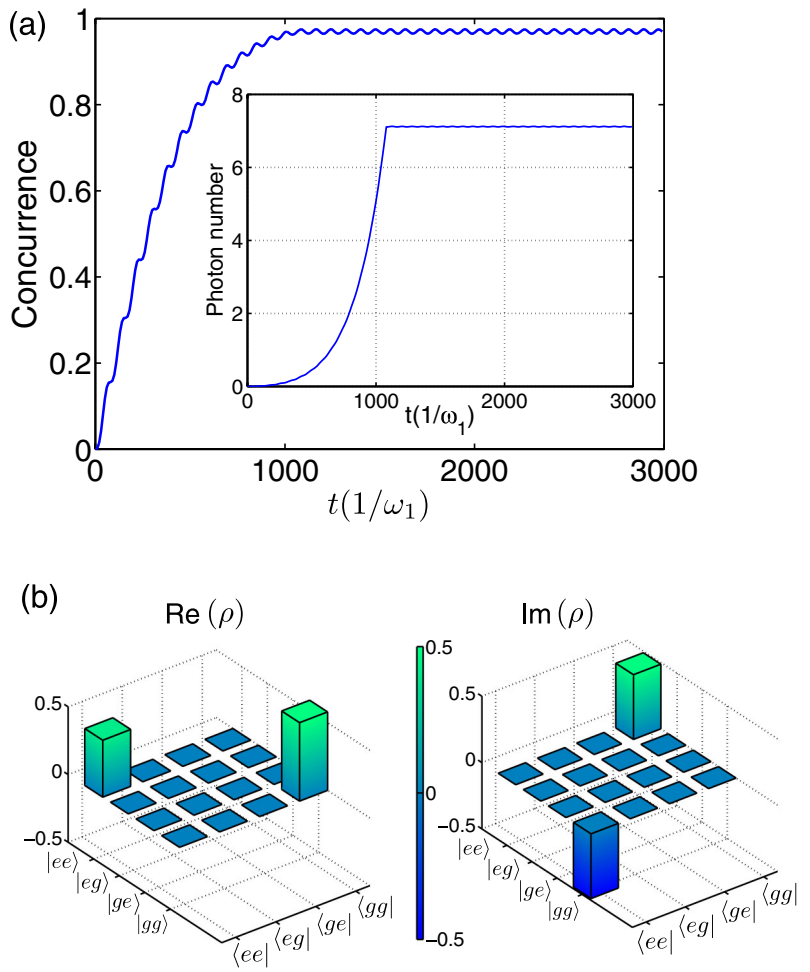

FIG. 3 (color online). (a) Concurrence and mean photon number as a function of time in units of the cavity frequency $\omega_{1}$. Here, the chosen parameters are $\omega_{1} / 2 \pi=4 \mathrm{GHz}$, $\omega_{2} / 2 \pi=5 \mathrm{GHz}$, the impedance for both cavities is $Z_{0}=50 \Omega$, and the critical current of the SQUID junctions is $I_{C}=1.1 \mu \mathrm{A}$. Such parameters result in a squeezing parameter $\alpha_{0}=\omega_{1} \times 10^{-3}$. Each qubit is resonant with its corresponding cavity and they are coupled with the same interaction strength $g=0.04 \omega_{2}$. (b) Real and imaginary parts of the density matrix $\rho$ associated with the two-qubit system. 


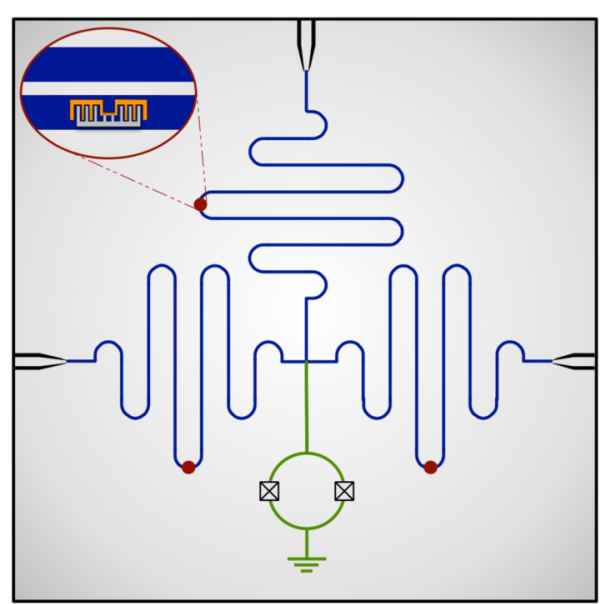

FIG. 4 (color online). Three coplanar waveguide resonators are connected to the ground through a SQUID. Each resonator is coupled with a resonant transmon qubit. This scheme allows generation of GHZ-like entangled states, through a first-order process. By using this circuit design as a building block, it is possible to explore more complex configurations and to build scalable cavity networks [21].

superconducting devices. This enables us to envision more complex configurations which generalize the concept of the dynamical Casimir effect to the multipartite case. Let us consider three resonators connected to the ground via a SQUID, as shown in Fig. 4. Injecting a fast-oscillating magnetic flux through the SQUID results in varying boundary conditions, which generate correlated photons pairs distributed in the three cavity modes. Such a configuration has no direct analogy with optical cavities, as opposed to the bipartite case. The Hamiltonian that describes the circuit of Fig. 4 is composed of three Jaynes-Cummings interactions and three time-dependent direct couplings between the field quadratures of each resonator pair

$$
\begin{aligned}
\mathcal{H}= & \hbar \sum_{\ell=1}^{3}\left[\omega_{\ell} a_{\ell}^{\dagger} a_{\ell}+\frac{\omega_{\ell}^{q}}{2} \sigma_{\ell}^{z}+g_{\ell}\left(\sigma_{\ell}^{+} a_{\ell}+\sigma_{\ell}^{-} a_{\ell}^{\dagger}\right)\right] \\
& +\hbar \sum_{\langle\ell, m\rangle} \alpha_{\ell m}(t)\left(a_{\ell}^{\dagger}+a_{\ell}\right)\left(a_{m}^{\dagger}+a_{m}\right) .
\end{aligned}
$$

If the external flux threading the SQUID is composed of three signals oscillating at the frequencies $\omega_{\ell m}^{d}=\omega_{\ell}+\omega_{m}$, we can isolate the two-mode squeezing terms as in Eq. (2).

Generating multipartite entanglement is a challenging task, since it requires multiqubit gates whose operation fidelity is considerably lower than the single- or two-qubit gates. Here we show that our protocol allows generation of genuine multipartite entanglement (GME). With GME, we refer to quantum correlations which cannot be described using mixtures of bipartite entangled states alone. The negativity [40] is an entanglement monotone that estimates the bipartite entanglement shared between two subsystems
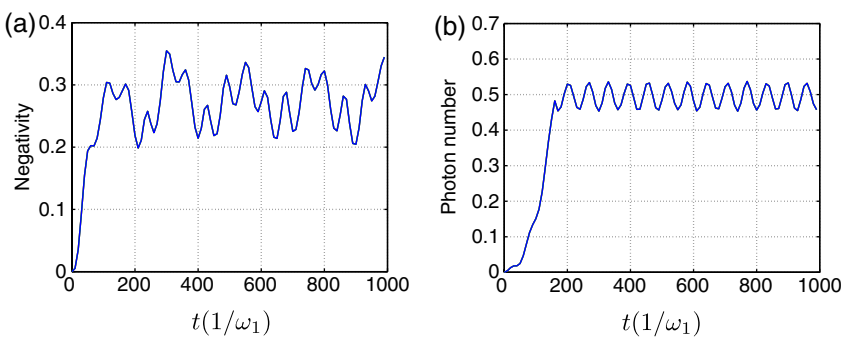

FIG. 5 (color online). (a) Negativity of the bipartite system obtained isolating one qubit from the set of the other two, as a function of time. Here, we considered resonator frequencies of $\omega_{1} / 2 \pi=3.8 \mathrm{GHz}, \omega_{2} / 2 \pi=5.1 \mathrm{GHz}$, and $\omega_{3} / 2 \pi=7.5 \mathrm{GHz}$. The SQUID is identical to the bipartite case and we use resonant qubits. The coupling parameters are homogeneous and their bare value is given by $\alpha_{0}=5 \omega_{1} \times 10^{-3}$. (b) Average photon number in each cavity as a function of time. Because of the symmetric configuration the photon distribution is the same for the three cavities.

of any possible bipartition; it ranges from zero for separable to $1 / 2$ for maximally entangled states. It is defined as $\mathcal{N}(\rho)=\left\|\rho^{T_{A}}\right\|_{1}-1 / 2$, where $\left\|\rho^{T_{A}}\right\|_{1}$ is the trace-norm of the partial transpose of the bipartite mixed state $\rho$. Numerical results on the negativity, shown in Fig. 5(a), indicate the generation of highly entangled states of three qubits. Figure 5(b) shows the average photon number in each cavity. In order to prove that such a state is not biseparable, we evaluate an entanglement monotone that detects only multipartite quantum correlations, called genuine multipartite entanglement (GME) concurrence $\mathcal{C}_{\mathrm{GME}}$. It is obtained after an optimization process over all decomposable witnesses $W=P+Q^{T_{A}}$, where $P$ and $Q$ are positive semidefinite [41,42]. Our results, $\max \left(\mathcal{C}_{\mathrm{GME}}\right) \approx 0.3$, confirm the existence of genuine multipartite entanglement.

Finally, to identify the entanglement class of three-qubit states, we make use of the entanglement witness [43] $\mathcal{W}_{\mathrm{GHZ}}=3 / 4 \llbracket-P_{\mathrm{GHZ}}, \quad$ where $\quad P_{\mathrm{GHZ}}=|\mathrm{GHZ}\rangle\langle\mathrm{GHZ}|$. Negative values for $\operatorname{Tr}\left[\rho \mathcal{W}_{\mathrm{GHZ}}\right]$ imply that for any decomposition $\rho=\sum_{j} p_{j} \rho_{j}$ at least one $\rho_{j}$ is a GHZ state, and so $\rho$ belongs to the GHZ class. Local operations do not change the entanglement class, it means the witness can be optimized by minimizing $\operatorname{Tr}\left[F \rho F^{\dagger} \mathcal{W}_{\mathrm{GHZ}}\right]$, where $F=F_{1} \otimes F_{2} \otimes F_{3}$, and $F_{i}$ are arbitrary single-qubit SLOCC operations. We obtained $\mathcal{W}_{\mathrm{GHZ}}=-0.06$, proving generation of (mixed) GHZ-like states, which belong to the most general entanglement class [20].

This scheme can be generalized to study entanglement generation in one- and two-dimensional cavity arrays in different geometries. Beyond the proposed model, our results show that superconducting circuit technology allows us to exploit the DCE physics as a useful resource for scalable quantum information protocols, generation of multipartite entanglement in artificial atoms, and as a building block for microwave quantum networks. 
We thank Géza Tóth and Jens Siewert for useful discussions. The authors acknowledge support from the Spanish MINECO FIS2012-36673-C03-02, Ramón y Cajal Grant No. RYC-2012-11391, UPV/EHU UFI 11/55, Basque Government IT472-10, CCQED, PROMISCE, SCALEQIT EU projects, and the Swedish Research Council.

[1] W. E. Lamb and R. C. Retherford, Phys. Rev. 72, 241 (1947).

[2] M. E. Peskin and D. V. Schroeder, An Introduction to Quantum Field Theory (Westview Press, Boulder, CO, 1995).

[3] H. B. G. Casimir, Proc. K. Ned. Akad. Wet. B 51, 793 (1948).

[4] S. K. Lamoreaux, Phys. Rev. Lett. 78, 5 (1997).

[5] U. Mohideen and A. Roy, Phys. Rev. Lett. 81, 4549 (1998).

[6] G. T. Moore, J. Math. Phys. (N.Y.) 11, 2679 (1970).

[7] C. M. Wilson, G. Johansson, A. Pourkabirian, M. Simoen, J. R. Johansson, T. Duty, F. Nori, and P. Delsing, Nature (London) 479, 376 (2011).

[8] P. Lähteenmäki, G. S. Paraoanu, J. Hassel, and P. J. Hakonen, Proc. Natl. Acad. Sci. U.S.A. 110, 4234 (2013).

[9] D. A. R. Dalvit and P. A. Maia Neto, Phys. Rev. Lett. 84, 798 (2000).

[10] P. A. Maia Neto and D. A. R. Dalvit, Phys. Rev. A 62 , 042103 (2000).

[11] N. B. Narozhny, A. M. Fedotov, and Yu. E. Lozovik, Phys. Rev. A 64, 053807 (2001).

[12] N. B. Narozhny, A. M. Fedotov, and Yu. E. Lozovik, Laser Phys. 13, 298 (2003).

[13] A. V. Dodonov, V. V. Dodonov, and S. S. Mizrahi, J. Phys. A 38, 683 (2005).

[14] M. A. Andreata and V. V. Dodonov, J. Opt. B 7, S11 (2005).

[15] A. V. Dodonov and V. V. Dodonov, Phys. Rev. A 85, 055805 (2012).

[16] M. A. Nielsen and I. L. Chuang, Quantum Computation and Quantum Information (Cambridge University Press, Cambridge, England, 2000)

[17] A. Blais, R. S. Huang, A. Wallraff, S. M. Girvin, and R. J. Schoelkopf, Phys. Rev. A 69, 062320 (2004).

[18] I. Chiorescu, P. Bertet, K. Semba, Y. Nakamura, C. J. P. M. Harmans, and J. E. Mooij, Nature (London) 431, 159 (2004).

[19] A. Wallraff, D. I. Schuster, A. Blais, L. Frunzio, R. S. Huang, J. Majer, S. Kumar, S. M. Girvin, and R. J. Schoelkopf, Nature (London) 431, 162 (2004).
[20] A. Acín, D. Bruß, M. Lewenstein, and A. Sanpera, Phys. Rev. Lett. 87, 040401 (2001).

[21] See Supplemental Material at http://link.aps.org/ supplemental/10.1103/PhysRevLett.113.093602, which contains the full derivation of the quantum model for the superconducting circuit and an outlook section and includes Refs. [22,23].

[22] K. K. Likharev, Dynamics of Josephson Junctions and Circuits (Gordon, Amsterdam, 1986).

[23] M. Wallquist, V. S. Shumeiko, and G. Wendin, Phys. Rev. B 74, 224506 (2006).

[24] M. J. Hartmann, F. G. S. L. Brando, and M. B. Plenio, Nat. Phys. 2, 849 (2006).

[25] T. D. Ladd, F. Jelezko, R. Laflamme, Y. Nakamura, C. Monroe, and J. L. OBrien, Nature (London) 464, 45 (2010).

[26] F. K. Wilhelm and J. Clarke, Nature (London) 453, 1031 (2008).

[27] M. Devoret and R. J. Schoelkopf, Science 339, 1169 (2013).

[28] V. Bouchiat, D. Vion, P. Joyez, D. Esteve, and M. H. Devoret, Phys. Scr. T76, 165 (1998).

[29] J. E. Mooij, T. P. Orlando, L. Levitov, L. Tian, C. H. van der Wal, and S. Lloyd, Science 285, 1036 (1999).

[30] J. M. Martinis, M. H. Devoret, and J. Clarke, Phys. Rev. Lett. 55, 1543 (1985)

[31] J. Koch, T. Yu, J. Gambetta, A. Houck, D. Schuster, J. Majer, A. Blais, M. Devoret, S. Girvin, and R. Schoelkopf, Phys. Rev. A 76, 042319 (2007).

[32] H. Paik et al., Phys. Rev. Lett. 107, 240501 (2011).

[33] J. B. Chang et al., Appl. Phys. Lett. 103, 012602 (2013).

[34] D. F. Bruschi, C. Sabín, P. Kok, G. Johansson, P. Delsing, and I. Fuentes, arXiv:1311.5619.

[35] T. P. Orlando and K. A. Delin, Foundations of Applied Superconductivity (Addison-Wesley, Reading, MA, 1991).

[36] M. Steffen, M. Ansmann, R. C. Bialczak, N. Katz, E. Lucero, R. McDermott, M. Neeley, E. M. Weig, A. N. Cleland, and J. M. Martinis, Science 313, 1423 (2006).

[37] L. DiCarlo et al., Nature (London) 460, 240 (2009).

[38] W. K. Wootters, Phys. Rev. Lett. 80, 2245 (1998).

[39] B. Kraus and J. I. Cirac, Phys. Rev. Lett. 92, 013602 (2004).

[40] G. Vidal and R. F. Werner, Phys. Rev. A 65, 032314 (2002).

[41] B. Jungnitsch, T. Moroder, and O. Gühne, Phys. Rev. Lett. 106, 190502 (2011).

[42] C. Eltschka and J. Siewert, Sci. Rep. 2, 942 (2012).

[43] N. Kiesel, C. Schmid, G. Tóth, E. Solano, and H. Weinfurter, Phys. Rev. Lett. 98, 063604 (2007). 